\title{
THE INTERACTION OF RARE GASES WITH METALS
}

\author{
G.G. Kleiman* \\ Research Laboratories, General Motors Corporation, Warren, MI 48090, U.S.A. \\ and \\ U. Landman
}

Institute for Fundamental Studies, Department of Physics and Astronomy, University of Rochester, Rochester, NY 14627, U.S.A.

(Received 2 October 1975 by A.A. Maradudin)

\begin{abstract}
Interaction potentials of rare gases with metal surfaces are calculated and compared with experimental data. A local dielectric function formalism and the density functional method are used in the calculation of the attractive and repulsive energies respectively. Depth and curvature variations of the potentials suggest a classification into light $(\mathrm{He}, \mathrm{Ne})$ and heavy rare gases. Sensitivity of the results to the model description of the metal surface electronic density is examined.
\end{abstract}

KNOWLEDGE of the weak forces of interaction of an atom or a molecule with a surface is fundamental to the understanding of physiorption phenomena ${ }^{\mathbf{1 , 2}}$ (distinguished from chemisorption on the basis of binding energy magnitudes; on the order of $\mathrm{mV}$ for the former and $\mathrm{eV}$ for the latter), to the evaluation of crosssections and scattering characteristics of elastic and inelastic atomic collisions with surfaces, ${ }^{3,4}$ to the calculations of thermodynamic properties of adsorption systems expresses as statistical mechanics integrals of functions of the gas-solid potential for isolated atoms 5 and to studies of critical phenomena in physisorption systems. $^{3,6}$

In a series of recent publications ${ }^{7-10}$ we discussed local and non-local theories of physisorption and calculated interaction potentials for He on a variety of metals. In this paper we extend the theory for the evaluation of interaction energies of other rare gases with metal surfaces. The results of our calculations suggest a classification of the rare gases, following their interaction characteristics (depth and curvature of the interaction potential), into light ( $\mathrm{He}, \mathrm{Ne}$ ) and heavy ( $\mathrm{Ar}, \mathrm{Kr}, \mathrm{Xe})$ rare gases. Examination of available scattering and adsorption data and preliminary calculations employing our potentials indicate that such a classification may apply to a large number of properties of rare gas - metal surface systems.

Physisorption is considered as a weak-coupling interaction which does not involve charge rearrangment

* Present address: Instituto de Fisica, Universidad Nacional Autonoma de Mexico, Apartado Postal 20-364, Mexico 20 D.F., Mexico. or other chemical transformation. The total interaction energy $U(d)$, of an atom separated from a surface by a distance $d$, is written as a sum of an attraction (Van der Waals) energy $E_{V w}(d)$, and a repulsion energy $E_{R}(d)$,

$$
U(d)=-E_{V W}(d)+E_{R}(d) .
$$

The Van der Waals energy, which contains exchange, correlation and electrostatic contributions, ${ }^{9}$ originates from the response of the atom to the random fluctuations of the electromagnetic field in the solid. The solid is considered here as a uniform continuous medium due to the long wavelengths of the field fluctuations. In previous studies $^{7-10}$ we emphasized the importance of an accurate description of the atomic frequency response in the evaluation of the interaction. While such information is relatively easy to obtain for $\mathrm{He}$, it is more difficult for the other rare gases. Consequently, in the following calculations we have used an approximate expression for the attractive interaction. In the local dielectric function formulation the Van der Waals energy can be written as

$$
\begin{aligned}
E_{V W}(d) & =\frac{\omega_{\mathrm{p}} / \sqrt{ } 2}{8 d^{3}} \int_{0}^{\infty} \mathrm{d} E f(E)\left[E\left(E+\omega_{p} / \sqrt{ } 2\right)\right]^{-1}, \\
f(E) & \equiv \sum_{\mathrm{j}} f_{j} \delta\left(E-\omega_{j}\right)+f_{c}(E),
\end{aligned}
$$

where $\omega_{p}$ is the plasma frequency of the metal substrate, $\omega_{j}$ and $f_{j}$ are discrete atomic transition frequencies and oscillator strengths respectively, and $f_{c}$ is the oscillator strength of transition to the continuum. Defining an "average" transition frequency 


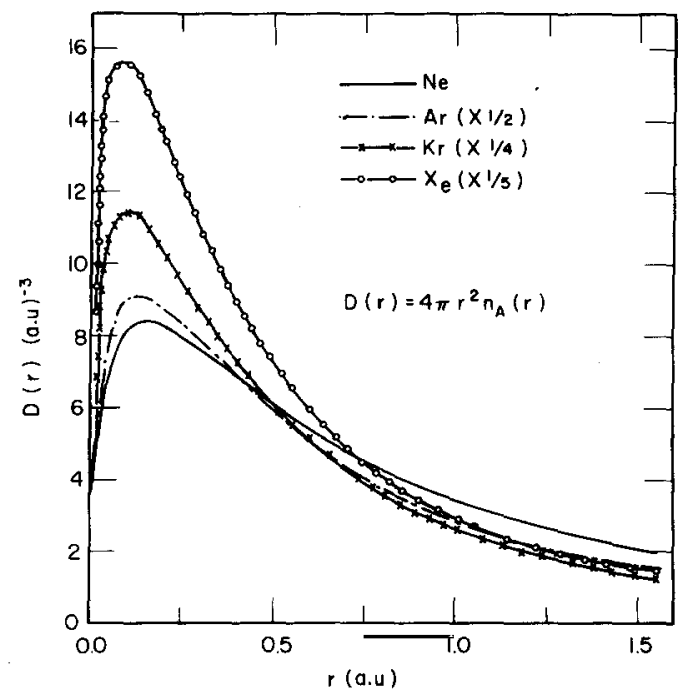

Fig. 1. Modified Thomas-Fermi radial distribution functions for $\mathrm{Ne}, \mathrm{Ar}, \mathrm{Kr}$, and $\mathrm{Xe}$.

$\omega_{Z}=\left(Z_{\text {eff }} / \alpha\right)^{1 / 2}$, where $\alpha$ is the static polarizability and $Z_{\text {eff }}$ the number of valence electrons for which the $f$-sum rule is essentially exhausted $\left(Z_{\text {eff }}=2\right.$ for He and 6 for the other rare gases ${ }^{12}$, a good approximation ${ }^{13}$ to the integral in equation ( 2 ) is given by

$$
E_{V W}(d)=\frac{Z_{\mathrm{eff}}\left(\omega_{p} / \sqrt{ } 2\right)}{8 d^{3} \omega_{Z}\left(\omega_{Z}+\omega_{p} / \sqrt{ } 2\right)}
$$

The repulsion energy is given in our model by the change in the electronic kinetic energy of the combined system upon the approach of the atom to the surface. Using the energy expression of the density functional formalism, expanded to first-order in a series of density gradients, ${ }^{14}$ yields an expression for the repulsion energy in terms of the electron densities of the isolated atom and metal surface. In the evaluation of the atomic charge density $n_{A}$, we have used the Thomas-Fermi potential function proposed by Latter ${ }^{15}$ with the modification due to March $^{16}$ to include exchange. The atomic charge density is given as

$$
n_{A}(x)=\lambda^{3} n_{A, T F}(x),
$$

where $x=r / \mu, \mu=0.8853 Z^{-1 / 3}$ and $Z$ is the atomic number. $\lambda$ is a variational parameter [see equation (5.14) in reference 16] and $n_{A, T F}$ is given by

$$
\begin{array}{rlrl}
n_{A, T F}(x) & =9.553 \times 10^{-2}(Z / \mu)^{3 / 2}[\phi(x) / x]^{3 / 2} \\
& =0 & & \text { for } Z \phi(x)>1 \\
& & \text { otherwise }
\end{array}
$$

where $\phi(x)$ is a universal function of $x$ [see reference 15 , equation (5)]. The radial charge distributions $[D(r)=$ $\left.4 \pi r^{2} n_{A}(r)\right]$ obtained from the above expressions, for $\mathrm{Ne}$, $\mathrm{Ar}, \mathrm{Kr}$ and $\mathrm{Xe}$ are shown in Fig. 1, (the cut-off radii

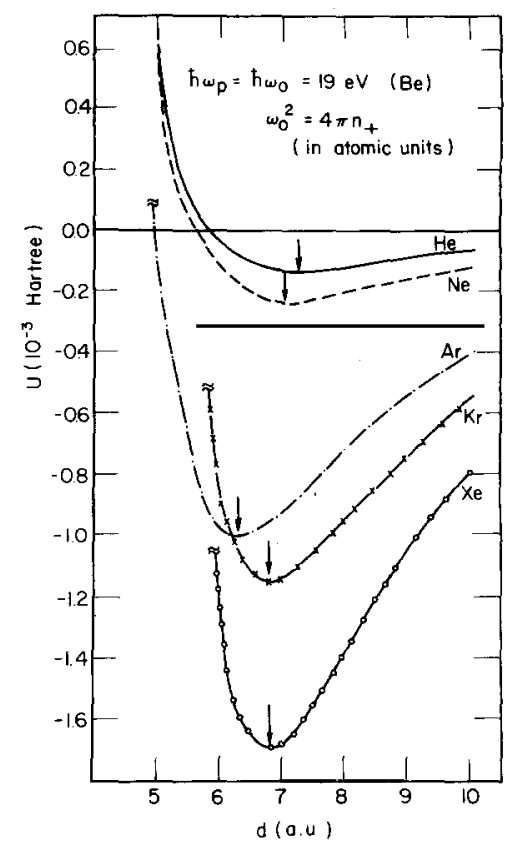

Fig. 2. Interaction potentials of rare gases with $\mathrm{Be}$ $\left(\hbar \omega_{p}=19 \mathrm{eV}\right)$. The He results were obtained as described in reference 9 . Values of the static atomic polarizabilities used in the calculations: $2.6424,10.9875$, 16.6161 , and $27.0306\left(a_{0}^{3}\right)$ for $\mathrm{Ne}, \mathrm{Ar}, \mathrm{Kr}$, and $\mathrm{Xe}$ respectively. An approximate model ${ }^{21}$ of the metal electronic density was used with $\beta=1.26 \mathrm{a} . u^{-1}$ Note variations in characteristics of the potentials between $\mathrm{He}$, $\mathrm{Ne}$ and $\mathrm{Ar}, \mathrm{Kr}$ and $\mathrm{Xe}$.

defined in equation (5) are: $1.726,2.094,2.494$ and 2.694 a.u., respectively).

The electron density at the surface region of a metal decreases rapidly oscillating inside the solid (Friedel oscillations) and spreading into the vacuum, decaying to zero over a distance of atomic dimension. ${ }^{17}$ Recent studies have demonstrated the sensitivity of surface properties (work function and surface energy ${ }^{17,18}$ and surface plasmons ${ }^{19,20}$, to the electron density profile at the surace region. In our previous calculations we have used an approximate form ${ }^{21}$ (extended ThomasFermi method) for the electron density, [see equation (3.15) in reference 9]. As discussed by Lang $^{\mathbf{1 8}}$ this approximation is expected to apply better to quantities whose values are obtained by an integration over the entire surface-region electron distribution. At distances typical to physisorption ( 5 a.u.) the atom-surface repulsion is expected to be sensitive mostly to details of the "vacuum tail" of the metal electron density.

Comparisons of calculated binding energies and equilibrium distances (see Table 1), where we have used the approximate and a self-consistent ${ }^{\mathbf{1 7}}$ electron densities demonstrate the sensitivity of our results to the description of the electron distribution at the surface region. 
Table 1. Binding energies and equilibrium distances for rare gases on metals

\begin{tabular}{|c|c|c|c|c|c|c|}
\hline System* & $\begin{array}{l}-U_{\exp } \\
\left(10^{-3} \text { Hartree }\right)\end{array}$ & Ref. & $\begin{array}{l}-U(\mathrm{sc}) \\
\left(10^{-3} \text { Hartree }\right)\end{array}$ & $\begin{array}{l}d_{e q} \\
\text { (a.u.) }\end{array}$ & $\begin{array}{l}-U \text { (nsc) } \\
\left(10^{-3} \text { Hartree }\right)\end{array}$ & $\begin{array}{l}d_{\text {eq }} \\
\text { (a.u.) }\end{array}$ \\
\hline $\mathrm{Ne} / \mathrm{Pt}$ & 0.526 & 26 & 0.398 & 6.0 & 0.287 & 6.9 \\
\hline $\mathrm{Ne} / \mathrm{Ag}$ & 0.351 & 33 & 0.462 & 5.4 & 0.392 & 6.0 \\
\hline $\mathrm{Ar} / \mathrm{Cu}$ & 3.332 & 26 & 1.863 & 4.7 & 1.303 & 5.7 \\
\hline $\operatorname{Ar} / Z_{n}$ & 2.503 & 26 & 2.984 & 3.0 & 1.710 & 5.2 \\
\hline $\mathrm{Ar} / \mathrm{Ni}$ & 1.103 & 34 & 1.830 & 5.1 & 1.194 & 6.2 \\
\hline $\mathrm{Ar} / \mathrm{W}$ & 2.869 & $28 \mathrm{a}$ & 1.713 & 5.3 & 1.092 & 6.4 \\
\hline $\mathrm{Ar} / \mathrm{Pt}$ & 2.105 & 26 & 1.857 & 5.2 & 1.223 & 6.1 \\
\hline $\mathrm{Kr} / \mathrm{Ag}$ & 2.232 & 31 & 2.490 & 4.8 & 1.964 & 5.6 \\
\hline $\mathrm{Kr} / \mathrm{Pt}$ & 3.364 & 26 & 1.960 & 5.7 & 1.378 & 6.6 \\
\hline
\end{tabular}

* Polarizabilities of the rare gases as in caption to Fig. 2. Values of the metal plasma frequencies $\left(\omega_{p}\right)$ and the variational parameters $(\beta)$ as given in Table 1 of reference 9.

Finally, anticipating the following discussion of our calculations, we should note that while qualitative characteristics and trends are predicted by our theory for the interaction with metal surfaces for atoms in the series $\mathrm{He}$ to Xe, we found it of quantitative value for the interaction of $\mathrm{He}, \mathrm{Ne}$ and $\mathrm{Ar}$ and in some cases $\mathrm{Kr}$. The chemical nature of $\mathrm{Xe}$ is well known. ${ }^{22}$ Considering the large polarizabilities and charge distributions of $\mathrm{Xe}$ and $\mathrm{Kr}$ it is expected that they will participate in an interaction of chemical nature with a metal substrate. Under these circumstances, weak coupling approximations are inadequate and a self-consistent treatment (or a variant thereof) of the combined atom-surface system is required.

\section{RESULTS AND DISCUSSION}

The method described above was used by us to calculate the interaction potentials between rare gases and a variety of metals. The results shown in Fig. 2 serve to illustrate some general characteristics of our results, (to facilitate comparison with our previous results for He physisorption, we used a non self-consistent metal electronic density). Evident from the results are the marked differences in magnitude and curvature of the potentials for $\mathrm{He}$ and $\mathrm{Ne}$ and those for $\mathrm{Ar}, \mathrm{Kr}$ and $\mathrm{Xe}$, which lead to their classification as light and heavy rare gases, respectively. The curvature of the potentials exhibits an increase in "stiffness" (a consequence of the repulsion) for the heavy gases compared to the light ones. These results indicate that the normal component of the potential-surface for the motion of an atom incident on a metal is rather shallow for the light gases, turning progressively deeper for the heavy gases. The classification of rare gas scattering from metals into a quasielastic, inelastic and trapping dominated regimes and the trends in the estimated trapping probabilities obtained from the experimental data, ${ }^{23}$ correlate with our results. The differences in the interaction characteristics of atoms in the two classes are reflected also in the eigenstate spectra derived from our calculated potentials. The above suggest the possibility of observing "bound state resonances" (selective adsorption ${ }^{24}$ ) in the elastic scattering intensity of rare gases from metals. Since the increase in the number of bound states and their density in the series $\mathrm{He}$ to $\mathrm{Xe}$, is accompanied by an increase in the probability of inelastic processes, the need for efficient velocity selectors in atom-surface scattering experiments is emphasized. ${ }^{25}$ Finally, we observe that contrary to the "sum-of-radii" rule, the atom-surface equilibrium distance decreases in the series $\mathrm{He}, \mathrm{Ne}, \mathrm{Ar}$ followed by a slight increase for $\mathrm{Kr}$ and $\mathrm{Xe}$, and is larger than the sum of radii of the gas and metal atoms. Note, that in the jellium construction the surface plane is located half an interplanar spacing in front of the outermost lattice plane, ${ }^{18}$ (see also discussion in reference 9 ).

In Table 1 we display results of our calculations, employing two models for the metal surface electronic density [non self-consistent ${ }^{21}$ (nsc), and self-consistent ${ }^{17}$ (sc)]. In comparing our results to available experimental data we should note that the most direct way of measurin physisorption binding energies is the calorimetric method, but even here a large estimated error of $\sim 40 \%$ is stated. ${ }^{26}$ The other experimental approaches which include: studies in field emission, ${ }^{27,28}$ atomic scattering $^{4,25}$ and desorption, ${ }^{29}$ determination of equation of state via work function and LEED intensity measurements, ${ }^{30}$ isotherms derived from ellipsometry data ${ }^{31}$ and time of flight measurements, ${ }^{32}$ all provide an indirect measure of the binding energies, yielding results which are susceptible to large errors. Finally, we remark on the sensitivity of the calculations to the model description of the metal surface and the overall good agreement between the theoretical values obtained by employing a selfconsistent electronic density and the experimental data. 
Acknowledgements - We thank Professor H. Saltsburg for helpful remarks. One of us (U.L) would like to thank Professor E.W. Montroll for his encouragement and interest.

\section{REFERENCES}

1. BOND G.C., Catalysis by Metals. Academic Press, NY (1962).

2. YOUNG D.M. \& CROWELL A.D., Physical Adsorption of Gases. Butterworth, London (1962).

3. HOBSON J.P., CRC Rev. (Solid State Sci.) 4 (2), 221 (1974).

4. LOGAN R.M., in Solid State Surface Science (Edited by GREEN M.) Vol. 3, p. 1. Marcel Dekker, NY (1973)

5. STEELE W.A., Surf. Sci. 36, 317 (1973).

6. DASH J.G. in Prog. Surf. Sci. (Edited by DAVISON S.G.) Vol. 4, (3), 95 (1974).

7. KLEIMAN G.G. \& LANDMAN U., Phys. Rev. Lett. 31, 707 (1973).

8. KLEIMAN G.G. \& LANDMAN U., Phys. Rev. Lett. 33, 524 (1974).

9. KLEIMAN G.G. \& LANDMAN U., Phys. Rev. B8, 5484 (1973).

10. LANDMAN U. \& KLEIMAN G.G., J. Vac. Sci. Technol. 12, 206 (1975).

11. LIFSHITZ E.M., Zh. Eksp. Teor. Fiz. 29, 94 (1955). [Sov. Phys. -JETP 2, 73 (1956)] ; DZYALOSHINSKII I.E., LIFSHITZ E.M. \& PITAEVASKII L.P., Adv. Phys. 10, 165 (1961).

12. EDGELL W.F., in Argon, Helium and the Rare Gases (Edited by COOK G.A.) Vol. 1, p. 152. Interscience, NY (1962).

13. A similar expression is given by MAVROYANIS C., Mol. Phys. 6, 593 (1963).

14. HOHENBERG P. \& KOHN W., Phys. Rev. 136, B864 (1964); KOHN W. \& SHAM L.J., ibid. 140, A1133 (1965).

15. LATTER R., Phys. Rev. 99, 510 (1955).

16. MARCH N.H., Adv. Phys. 6, 1 (1957).

17. LANG N.D. \& KOHN W., Phys. Rev. B1, 4555 (1970).

18. LANG N.D. in Solid State Physics (Edited by SEITZ F., TURNBULL D. \& EHRENREICH H.) Vol. 28, p. 225. Academic Press, NY (1973).

19. BENNET A.J., Phys. Rev. B1, 203 (1970).

20. FEIBELMAN P.J., Phys. Rev. Lett. 30, 975 (1973).

21. SMITH J.R., Phys. Rev. 181, 522 (1969).

22. Noble Gas Compounds (Edited by HYMAN H.H.) University of Chicago Press (1963); HALL J.R., in Physical Chemistry (Edited by HENDERSON D.) Vol. 4, o. 159. Academic Press, NY (1970).

23. WEINBERG W.H. \& MERRILL R.P., J. Chem. Phys. 56, 2881 (1972).

24. LENNARD-JONES J.E. \& DEVONSHIRE A.F.,;Nature, Lond. 137, 1069 (1936).

25. For general reviews see: SALTSBURG H., Ann. Rev. Phys. Chem. 24, 493 (1973); TOENNIES J.P., Appl. Phys. 3, 91 (1974) and reference 4.

26. CHON H., FISHER R.A., McCAMMON R.D. \& ASTON J.G., J. Chem. Phys. 36, 1378 (1962).

27. EHRLICH G. \& HUDDA F.G., J. Chem. Phys. 30, 493 (1959).

28. (a) GOMER R., Aust. J. Phys. 13, 391 (1960); (b) ENGET T. \& GOMER R., J. Chem. Phys. 52, 5572 (1970).

29. YATES J.T. \& MADEY T.E., Surf. Sci. 28, 437 (1971).

30. PALMBERG P.W., Surf. Sci. 25, 598 (1971); see also TRACY J.C. \& PALMBERG P.W., ibid. 14, 274 (1969).

31. STEIGER R.F., MORABITO J.M., SOMORJAI G.A. \& MULLER R.H., Surf. Sci. 14, 299 (1969).

32. POLLOCK F., LOGAN H., HOBGOOD H. \& DAUNT J.G., Phys. Rev. Lett. 28, 346 (1972).

33. SAU R. \& MERRILL R.P., Surf. Sci. 34, 268, Table 1 (1973).

34. GUNDRY P.M. \& TOMPKINS F.C., i iuris. Faraday Soc. 56, 846 (196(1). 